\title{
A High-Performance Computing Framework for Analyzing the Economic Impacts of Wind Correlation
}

\author{
Cosmin G. Petra ${ }^{1, *}$, Victor M. Zavala ${ }^{2, *}$, Elias D. Nino-Ruiz ${ }^{3, *}$, Mihai \\ Anitescu ${ }^{4, *}$
}

Preprint ANL/MCS-P5148-0614

\begin{abstract}
We analyze the impact of capturing spatiotemporal correlations between wind supply points on grid dispatch procedures. We first show analytically that over/underestimation of correlation leads to nonintuitive cost biases. A detailed computational study for the U.S. state of Illinois grid reveals similar conclusions. Our computing framework combines a stochastic dispatch formulation with correlation information derived from a Rao-Blackwell-Ledoit-Wolf estimator. The estimator approximates the covariance matrix from a small number of expensive wind ensembles generated with a numerical weather prediction model. The prediction model is validated with real meteorological data to obtain realistic correlation information. The resulting stochastic dispatch problems are solved with the parallel interior-point solver PIPS-IPM on the BlueGene/Q (Mira) supercomputer at Argonne National Laboratory. We use the correlation information to generate a larger set of scenarios and implement a fast inference analysis capability to assess the accuracy of the system cost. We demonstrate that the computing framework enables sophisticated system-wide analyses that can be performed in minutes (as opposed to days if computations would be performed in serial). We find that strong and persistent biases result

\footnotetext{
*corresponding author

${ }^{1}$ Center for Applied Scientific Computing, Lawrence Livermore National Laboratory, Livermore, CA 94550, USA. E-mail: petra1@llnl.gov

${ }^{2}$ Department of Chemical and Biological Engineering, University of Wisconsin-Madison, Madison, WI 53706, USA. E-mail: victor.zavala@wisc.edu

${ }^{3}$ Department of Computer Science, Universidad del Norte, Barranquilla, ATL, Colombia. E-mail: enino@uninorte.edu.co

${ }^{4}$ Mathematics and Computer Science Division, Argonne National Laboratory, Argonne, IL 60439, USA. E-mail: anitescu@mcs.anl.gov.
} 
from neglecting correlations and conclude that coordinating uncertainty characterizations for wind power producers is necessary. The framework is also used to quickly assess the impact of operating the grid under different correlation strengths. Our work seeks to highlight the scope of modern high-performance computing systems and computational optimization tools.

Keywords:

covariance estimation, correlation, economic dispatch, stochastic optimization, wind power.

\section{Introduction}

Achieving efficient grid operations in the presence of intermittent renewable power is a challenge because these supply sources follow complex spatiotemporal patterns (see Fig. 1) that extend over wide geographical regions (e.g., tens to hundreds of kilometers) and long periods of time (i.e., hours to days). Reserve allocation procedures therefore can be ineffective, and more adaptive and systematic approaches based on stochastic and robust optimization techniques are needed.

Stochastic and robust optimization techniques rely on uncertainty characterizations. Correlation (or covariance) information, in particular, is key because this guides forecast aggregation/disaggregation procedures and because it is needed to characterize risk in dispatch cost and revenues of market players. For instance, if the supply of a wind farm in a region is uncorrelated from that in another region, these can be forecasted independently without affecting dispatch cost. When correlations exist, however, one would expect that

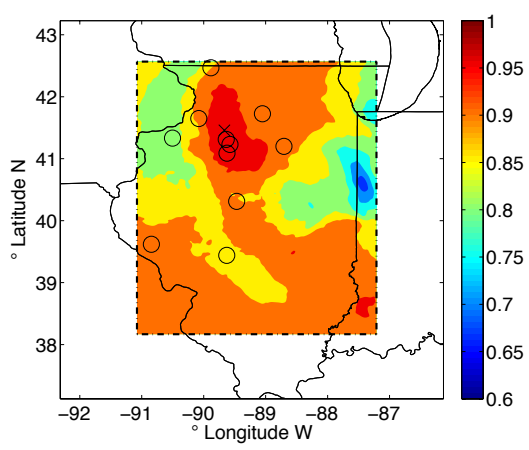

Figure 1: Spatial correlations for wind speed in the State of Illinois with respect to the location indicated by " $\times$ ". The locations indicated by circles depict actual wind farms. using independent forecasts will introduce errors in the uncertainty characterization and this will bias dispatch cost and shift incentives of the players (wind power suppliers, distributors, and consumers). 
This situation was hypothesized in the stochastic economic dispatch setting of Pritchard and coworkers [1] (see Section 5). In Xie et al. [2] such biases seem to occur in day-ahead reliability unit commitment and robust look-ahead economic dispatch models because of differences in the correlations given by different estimation techniques. Also, Ghofrani et al. [3] identify wind correlation as a determining factor in choosing wind sites and find empirically that it considerably impacts the reserve requirements and network congestion. The present study is, to the best of our knowledge, the first to address the issue of such cost biases and to offer a magnitude of the cost gaps that occur in realistically sized economic dispatch systems.

From a market implementation point of view, determining long-range correlations is a challenge because wind farm or solar power plant owners might not be willing to share their forecasting procedures and their site information with other markets players and the ISO. Consequently, they might prefer to construct their own forecasts and uncertainty levels, possibly neglecting correlations with other sites. Owners might also need to ignore long-range correlations because of computational limitations faced by their forecasting vendors. Computational challenges arise because properly resolving the space-time features of numerical weather prediction (NWP) systems requires significant computational power [4], and few computing sites exist in the world that can obtain forecasts that accurately capture both short-range conditions at the supply site and long-range behavior. When trying to properly characterize uncertainty and its correlation structure this points to the need both for a wide-area forecasting approach and for accommodating computational limitations.

Properly designing decentralized markets that factor in uncertainty in weatherdriven supply is necessary, but this requires significantly more complex information exchange mechanisms between the ISO and market players compared with existing deterministic settings $[1,5]$. To design such information exchange mechanisms, one needs to understand the effects that certain information has on performance. In this work, we study the effect of long-range correlation information on dispatch cost in the presence of wind power. We first present an analytic example to prove that correlation between suppliers' output can positively or negatively bias dispatch cost in non-intuitive ways (depending on the correlation direction). We then perform a detailed computational study using a stochastic economic dispatch setting in the Illinois transmission system. We use validated wind speed ensemble forecasts obtained with the NWP system WRF that are propagated through typical wind power curves in order to obtain wind power ensembles. We use the Rao-Blackwell-Ledoit-Wolf estimator 
to generate scenarios from the limited number of WRF samples available to perform inference analysis and refine the cost estimates.

Our computational study reveals that significant dispatch cost biases that would scale up to an order of hundreds of millions of dollars a year (if similar correlation patterns across the year hold) can be introduced by ignoring long-range correlation. We also show that confidence levels of dispatch cost differ significantly from the actual ones when correlations are neglected. In our study, the confidence intervals when ignoring correlations were narrower, thus underestimating the number of scenarios necessary to close the cost gap due to stochastic approximation of the wind uncertainty. From a market design perspective our study thus indicates that, as hypothesized in the stochastic market setting of [1], centralized forecasts that can properly account for correlations are superior to localized ones when used for constructing wind power bids by suppliers in markets with significant wind power penetration. This points to the need of coordinating forecasts and uncertainty characterizations in order to capture long-range correlations among power producers. Neglecting such correlations (e.g., by allowing producers to provide their own uncertainty characterizations) can introduce cost and price biases.

Diverse studies on the impact of wind correlation on power system operations have been reported in the literature. In [6] the authors propose a market clearing model and find that spatial correlations can have significant effects on locational marginal prices. In [7] the authors find that spatio-temporal correlations can drastically increase risk to consumers if wind farms are not dispersed enough. In [8] the authors propose a stochastic optimal power flow conclude that correlations can also alter reactive power. The studies available in the literature use simplified case studies based on IEEE test models and historical data sets. To the best of our knowledge, the analysis proposed in this work is the most sophisticated study reported in the literature on the effects of wind power correlation. In particular, our analysis combines advanced numerical weather prediction models (that have been validated with meteorological information), large-scale power system network models, stochastic market clearing formulations, parallel optimization solvers, advanced covariance estimation schemes, and inference analysis techniques. We combine these tools in a highperformance computing platform to enable sophisticated analyzes in minutes. Performing such analyzes on a standard serial computer would have taken days. Our work seeks to highlight the scope of modern high-performance computing systems and computational optimization tools.

The paper is structured as follows. In Section 2 we present a motivating 
example to illustrate the effect of correlation information on dispatch cost. In Section 3 we present a detailed computational study using data for the state of Illinois transmission system. This section describes the dispatch model, the scenario generation procedure, the covariance estimator, and the numerical results. Section 4 presents concluding remarks.

\section{Motivating Analytical Example}

Consider a single-node system with three suppliers and one consumer. Two suppliers, $G_{1}$ and $G_{2}$, have uncertain power output and the outputs follow Gaussian distributions, $\mathcal{N}\left(w_{1}, \sigma_{1}\right)$ and $\mathcal{N}\left(w_{2}, \sigma_{2}\right)$. We define $\rho \in[-1,1]$ as the correlation coefficient and assume that both suppliers have a cost $p_{w}$. The third supplier, $G_{3}$, is assumed to be deterministic, supplies power at cost $p_{t h}$ with $p_{t h}>p_{w}$ and has infinite capacity. The demand quantity $d$, is assumed to be deterministic and inelastic.

By construction, one can deduce that as much cheap power as possible should be produced. If this does not satisfy all demand, then $G_{3}$ will be dispatched to fulfill the remaining demand. Consequently, the negative dispatch cost is $c_{d}=\mathbb{E}\left[p_{w} \min \left(X_{1}+X_{2}, d\right)+p_{t h} \max \left(d-X_{1}-X_{2}, 0\right)\right]$. Furthermore, to derive the dependence $c_{d}=c_{d}(\rho)$, we write

$$
\begin{aligned}
c_{d} & =\mathbb{E}\left[p_{w} d+p_{w} \min \left(X_{1}+X_{2}-d, 0\right)+p_{t h} \max \left(d-X_{1}-X_{2}, 0\right)\right] \\
& =p_{w} d+\mathbb{E}\left[-p_{w} \max \left(d-X_{1}-X_{2}, 0\right)+p_{t h} \max \left(d-X_{1}-X_{2}, 0\right)\right] \\
& =p_{w} d+\mathbb{E}\left[\left(p_{t h}-p_{w}\right) \max \left(d-X_{1}-X_{2}, 0\right)\right] \\
& =p_{w} d+\left(p_{t h}-p_{w}\right) \mathbb{E}\left[d-\left(X_{1}+X_{2}\right) \mid X_{1}+X_{2} \leq d\right] .
\end{aligned}
$$

Here, $\mathbb{E}[X \mid Y]$ denotes the expectation of $X$ conditional on event $Y$. In an engineering context, this conditional expectation is given by the net load (which is random) subject to the condition that the dispatched generation is less than the load. Furthermore, since the random variable $X=X_{1}+X_{2}$ is normally distributed, $X \sim \mathcal{N}(\mu, \sigma)$, where

$$
\sigma(\rho)=\sqrt{\sigma_{1}^{2}+2 \rho \sigma_{1} \sigma_{2}+\sigma_{2}^{2}} \text { and } \mu=w_{1}+w_{2}
$$

$c_{d}$ can be expressed as

$$
\begin{aligned}
c_{d} & =p_{w} d+\left(p_{t h}-p_{w}\right) \mathbb{E}[d-X \mid X \leq d] \\
& =p_{w} d+\left(p_{t h}-p_{w}\right) \cdot d \cdot \Phi(d, \sigma)-\left(p_{t h}-p_{w}\right) \mathbb{E}[X \mid X \leq d],
\end{aligned}
$$


where $\Phi$ is the cumulative density function of $X$,

$$
\Phi(x, \sigma)=\frac{1}{2}\left[1+\operatorname{erf}\left(\frac{x-\mu}{\sqrt{2} \sigma}\right)\right] .
$$

We denote the probability density function of $X$ by $\phi(x, \sigma)=\frac{1}{\sqrt{2 \pi} \sigma} \exp \left(-\frac{(x-\mu)^{2}}{2 \sigma^{2}}\right)$. One can show (see Lemma 1 from the Appendix) that

$$
\mathbb{E}[X \mid X \leq d]=-\sigma^{2} \phi(d, \sigma)+\mu \Phi(d, \sigma) .
$$

Combining this equation with (2), we obtain

$$
c_{d}=p_{w} d+\left(p_{t h}-p_{w}\right)\left((d-\mu) \Phi(d, \sigma)+\sigma^{2} \phi(d, \sigma)\right) .
$$

In an engineering context, this indicates that we can express the dispatch cost as a function of the demand and the statistics of the random generation parameterized in the standard deviation $\sigma$ which is an implicit function of the correlation coefficient $\rho$ between the output of the two random generators. The dependence of the dispatch cost $c_{d}$ on this correlation parameter $\rho$ becomes clear and we have the following result:

Proposition 1. The dispatch cost $c_{d}(\rho)$ given by (5) is a strictly increasing function of the correlation parameter $\rho$.

Proof: See the Appendix.

This result shows that there can be a positive or negative bias (depending on the correlation direction) between dispatch cost values computed from different correlation structures (different uncertainty characterizations). This can be interpreted as a dispatch cost bias introduced by an error in the correlation coefficient. Such an error in the correlation coefficient can be the result of neglecting long-range information or improperly capturing it due to limitations of the NWP model and/or of the uncertainty quantification procedure [4].

Fig. 2 shows how dispatch cost depends on the correlation coefficient. For this $c_{d}$ is plotted as a function of $d$ for $\rho=\{-1,0,1\}$. We used the following parameters: $w_{1}=w_{2}=10 \mathrm{MWh}, \sigma_{1}=\sigma_{2}=2 \mathrm{MWh}, p_{t h}=50 \mathrm{USD} / \mathrm{MWh}$ and $p_{w}=5 \mathrm{USD} / \mathrm{MWh}$. With increasing $\rho$, the probability distribution of the total available wind $X=X_{1}+X_{2}$ exhibits an increasing variance (see (1)). Consequently, it is less likely to satisfy demand using the cheap uncertain supply 


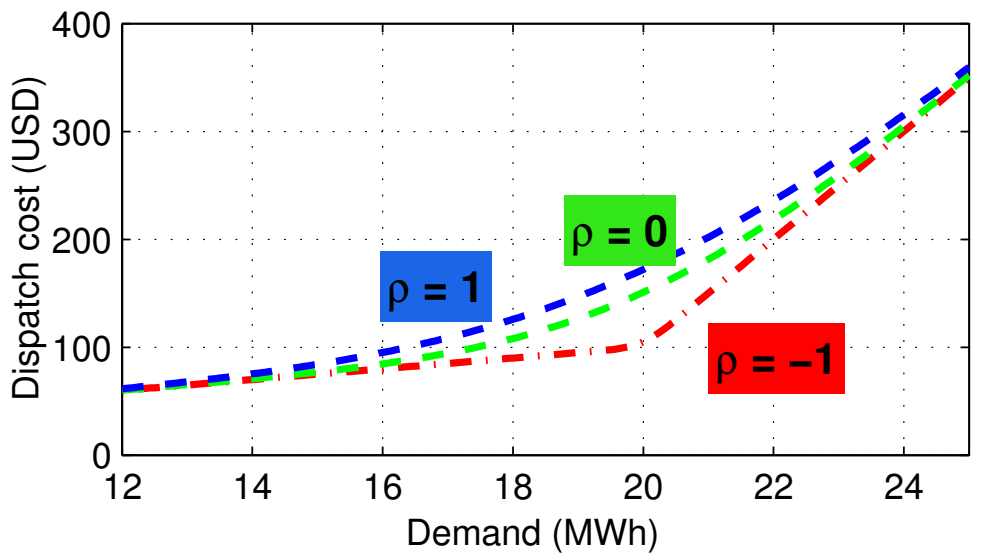

Figure 2: Dispatch cost $c_{d}$ as a function of demand $d$ for negative correlation $(\rho=-1)$, no correlation $(\rho=0)$, and positive correlation $(\rho=1)$.

and more likely to dispatch the more expensive supply, thus resulting in a higher cost. For instance, if two suppliers are fully positively correlated $(\rho=1)$, a low output of one will result in a low output of the other. In the extreme case of full negative correlation $(\rho=-1)$ cheap uncertain supply will most likely be used because if the output of one wind farm drops, then this implies that the output of the other one increases, so at least one wind farm is always active. From Fig. 2 we note also that, at low and high demands, correlation has less effect on the dispatch cost.

From a purely dispatch cost perspective, one can argue that an inaccurate forecasting system that overestimates and underestimates the correlations may lead to the same expected dispatch cost in the long run, compared with that obtained with an ideal forecasting system. While we do not refute this possibility, we point out that inaccurate forecasting also results in gaps in prices and revenues and, hence in market inefficiencies. These inefficiencies will bias incentives toward certain players in unpredictable ways that might not average out in the long run.

Errors in correlation between uncertain suppliers can occur for various reasons. For example, the owners of the wind farms might submit their own forecasts and scenarios, which the ISO will treat independently when dispatching. Such a situation is discussed in the stochastic market setting of [1]. Alternatively, correlations or the covariance matrix may be poorly estimated in the NWP systems because of the prohibitive computational cost of accurate estimators. Hence, we advocate the use of ISO-centric weather forecasting systems while mapping to wind power forecasts (that capture local and wind turbine ef- 
fects) can be done internally by wind farm owners in order to prevent disclosure of information. The numerical simulations of the next section indicate that savings of more than 10,000 USD per dispatch hourly period can be obtained for full-scale power grids, such as the one of the State of Illinois. The savings over one year can reach a hundred million dollars, assuming a comparable amount of savings per dispatch period in the rest of the year.

\section{High-Performance Computing Platform}

In this section, we present a detailed computational study to analyze the effects of correlation on dispatch cost and prices. The framework combines stochastic dispatch models, numerical weather prediction forecasts validated with real meteorological data, as well as covariance estimation, scenario generation, and inference analysis techniques. Our setting includes realistic data for the Illinois transmission system, and we assume a wind adoption scenario of $17 \%$. We emphasize that the objective of the following computational study is to provide insight on the type of behavior that can arise when correlation information is ignored and not to provide final numerical estimates.

\subsection{Stochastic Dispatch Model}

In our analysis we use the stochastic dispatch model from [1] that captures forward and spot components. The model assumes that the market is cleared at the current time using predictions of the uncertain conditions at the next time period. When uncertainty is realized, the suppliers are allowed to sell additional power or buy back under the realized conditions. The formulation is given by:

$$
\begin{array}{ll}
\min _{x_{i}, X_{i}(\omega)} & \sum_{i \in \mathcal{G}}\left(p_{i} x_{i}+\mathbb{E}_{\omega}\left[p_{i}^{+}\left(X_{i}(\omega)-x_{i}\right)_{+}-p_{i}^{-}\left(X_{i}(\omega)-x_{i}\right)_{-}\right]\right) \\
\text {s.t. } & \tau_{n}(f)+\sum_{i \in \mathcal{G}(n)} x_{i}=d_{n}, n \in \mathcal{N} \\
& \tau_{n}(F(\omega))-\tau_{n}(f)+\sum_{i \in \mathcal{G}(n)}\left(X_{i}(\omega)-x_{i}\right)=0, \quad n \in \mathcal{N}, \omega \in \Omega \\
& f, F(\omega) \in \mathcal{U}, \omega \in \Omega \\
& \left(x_{i}, X_{i}(\omega)\right) \in \mathcal{C}_{i}(\omega), \quad i \in \mathcal{G}, \omega \in \Omega
\end{array}
$$

Here, $\mathcal{N}$ denotes the set of nodes (buses) and $\mathcal{L}$ the set of transmission lines. The set of all suppliers is denoted by $\mathcal{G}$. Subsets $\mathcal{G}(n)$ denote the set of players 
connected to node $n$. The forward dispatched quantities for players are $x_{i}$, and the spot quantities under scenario $\omega$ are $X_{i}(\omega)$. The forward power flow through line $\ell \in \mathcal{L}$ is denoted by $f_{\ell}$, and $f$ denotes the vector of all line flows. Similarly, $F(\omega)$ denotes the vector of line flows $F_{\ell}(\omega)$ for each scenario $\omega$. The demand is assumed to be deterministic and perfectly inelastic and is represented by $d_{n}, n \in N$. The scenarios $\omega$ characterize the randomness in the model due to unpredictable capacities and are mathematically expressed as random vectors defined on some probability space $(\Omega, \mathcal{F}, P)$. The expectation $\mathbb{E}_{\omega}$ is taken with respect to the measure $P$. In practice, one considers a finite approximation of $\Omega$ obtained through sampling.

The objective consists of minimizing the forward dispatch cost $\sum_{i \in \mathcal{G}} p_{i} x_{i}$ plus the expected adjustment or recourse dispatch cost

$$
\sum_{i \in \mathcal{G}} \mathbb{E}_{\omega}\left[p_{i}^{+}\left(X_{i}(\omega)-x_{i}\right)_{+}-p_{i}^{-}\left(X_{i}(\omega)-x_{i}\right)_{-}\right]
$$

specific to individual scenarios. Here $[y]_{+}=\max \{y, 0\}$ and $[y]_{-}=\max \{-y, 0\}$. The coefficients $p_{i}$ denote the bid price, and $p_{i}^{+}$and $p_{i}^{-}$are price bids for realtime corrections of the generators. A supplier $i$ asks $p_{i}^{+}>p_{i}$ to sell additional power or asks $p_{i}^{-}<p_{i}$ to buy power from the system (e.g., reduces output). In our model we have used the prices $p_{i}^{-}=0.8 p_{i}$ and $p_{i}^{+}=1.2 p_{i}$. We highlight that this assumption implies that corrections from forward quantities are penalized symmetrically. In this symmetric case, the cost function reduces to:

$$
\sum_{i \in \mathcal{G}} p_{i} \mathbb{E}_{\omega}\left[X_{i}(\omega)\right]+\Delta p_{i} \mathbb{E}_{\omega}\left[\left|X_{i}(\omega)-x_{i}\right|\right]
$$

where $\Delta p_{i}=p_{i}^{+}-p_{i}=p_{i}-p_{i}^{-}$. This property was noticed by Pritchard and coworkers [1] and provides an interesting interpretation of the dispatch model. In particular, note that the expected value of the spot quantities $\mathbb{E}_{\omega}\left[X_{i}(\omega)\right]$ is penalized by the forward price $p_{i}$ and the expected corrections by the incremental price $\Delta p_{i}$. This indicates that the expected spot quantities $\mathbb{E}_{\omega}\left[X_{i}(\omega)\right]$ act as a forecast of the forward quantities $x_{i}$. This property is important because it indicates that the dispatch model averages spot behavior. Note also that (7) indicates that $\Delta p_{i}$ should be strictly greater than zero because, otherwise, solution multiplicity will exist. Although the symmetric case interpretation is interesting, we do acknowledge and warn the reader that assuming symmetry can have an effect on the numerical results of our study. We also highlight that we prefer to use symmetric offer prices as opposed to randomly generated offer prices in order to let wind generation be the only random effect affecting the system and thus have a more transparent analysis. 
The decision variables are the dispatch quantities for each generator, $x_{i}$, $X_{i}(\omega)$ and the power flows $f_{\ell}$ and $F(\omega)$. The forward dispatches $x_{i}$ are "ahead" decisions that account for randomness; the spot re-dispatches $X_{i}(\omega)$ represent "real-time" decisions that are appropriate corrections once an individual realization $\omega$ of the randomness is observed.

Function $\tau_{n}(\cdot)$ is a mapping of the flow vector to the node $n$. We denote by $\nu_{1}(n)$ the inflow lines into node $n \in N$ and by $\nu_{0}(n)$ the outflow lines. Equation (6a) describes the power flow through a node $n \in N$ which is the sum of power $\tau_{n}(f)=\sum_{\ell \in \nu_{1}(n)} f_{\ell}-\sum_{\ell \in \nu_{0}(n)} f_{\ell}$ imported via the transmission lines to node $n$ and power $\sum_{i \in \mathcal{G}(n)} x_{i}$ produced at node $n$. Equation (6b) is the secondstage correspondent of (6a), enforcing power flow balance at each node for each scenario $\omega$. It is shown in [1] that the multipliers associated with this "residual" formulation (not with the simpler equivalent form, $\tau_{n}(F(\omega))+\sum_{i \in \mathcal{G}(n)} X_{i}(\omega)=$ $0)$ gives the locational marginal prices to be used in the spot (or real-time) market. Equation (6c) represents maximum flow constraints on individual lines, with $\mathcal{U}$ usually being a polyhedron.

Equation (6d) expresses constraints on supply that come from technological limits of the generators (such as maximum/minimum capacity, limited rampup/down power on short notice) and intermittent availability of energy of some generators. In our model, $\mathcal{C}_{i}$ is deterministic for thermal generators (natural gas, coal, heater oil and nuclear) and given by $\mathcal{C}_{i}=\left\{\left(x_{i}, X_{i}\right): x_{i}, X_{i} \in\left[m_{i}, M_{i}\right], \mid x_{i}-\right.$ $\left.X_{i} \mid \leq r_{i}\right\}$, expressing capacity and ramp constraints. The uncertain output is modeled by $\mathcal{C}_{i}(\omega)=\left\{\left(x_{i}, X_{i}(\omega)\right): x_{i} \in\left[0, M_{i}\right], X_{i}(\omega) \in\left[0, W_{i}(\omega)\right]\right\}$, showing that the forward dispatch $x_{i}$ can be allowed to reach maximum installed capacity and the spot dispatch $X_{i}(\omega)$ can only be allowed to reach maximum power generated under scenario $\omega$. We consider ramp constraints with only one time step. In other words, the sets $\mathcal{C}_{i}$ and $\mathcal{C}_{i}(\omega)$ together with the network flow equations comprise the feasible set of the optimization problem. The above stochastic model seeks to dispatch generators in advance by hedging against uncertainty in the capacities of some generators (e.g., due to lack of knowledge on the wind conditions). The generator outputs are allowed to be corrected once uncertainty is revealed. The generator dispatch decisions respect DC power flow equations.

Our model was set up for the State of Illinois power grid which comprises 2,522 lines, 1,908 buses, 870 load buses and 225 generators. To obtain a large wind power installed capacity (approximately 17\%), we needed to create synthetic wind farms in addition to the existing ones. The synthetic farms were chosen to replace some existing coal or gas generators. This approach was taken 
specifically to avoid possible network congestion that would limit the amount of real wind adoption. In addition, we replaced only thermal generators that were mirrored by other (usually identically) generators in order to ensure that enough thermal generation was available to satisfy demand in the case of lowwind scenarios. The generation cost for the wind farm was set to 5USD/MWh, the lowest across all generators.

We highlight that the market model used in this study does not match existing deterministic markets. Designing an appropriate market that prices electricity under uncertainty is an active subject of research. The model used in our study is the more realistic model that we have found in the literature because it enables consistency between forward and spot decisions and it has desirable pricing and revenue properties $[1,5]$.

We also highlight that, while robust optimization is practical and more tractable approach to analyze wind correlation effects, this approach has to resort to a closed form representation (an approximation) of the uncertainty space. This is not straightforward to do in applications like wind forecasting where complex (non-Gaussian) and large spatio-temporal spaces need to be used and where uncertainty is often only available in the form of ensembles. Stochastic programming allows for a more direct use of of uncertainty information of this form and has some desirable pricing properties.

\subsection{Wind Scenario Generation}
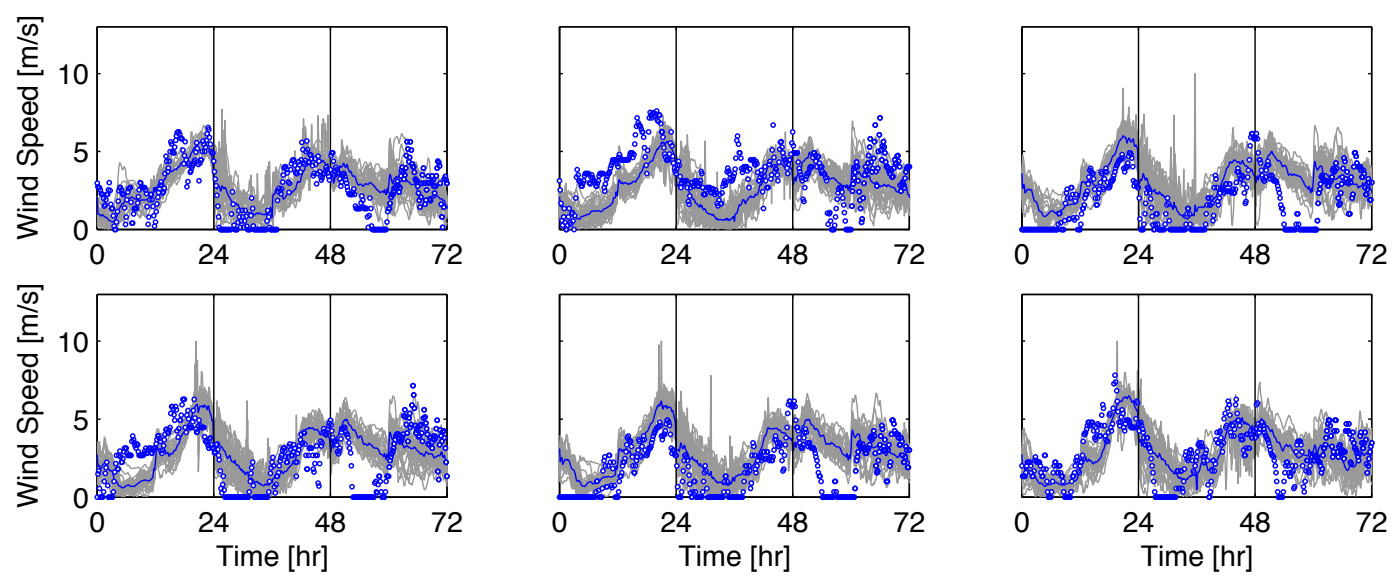

Figure 3: Wind speed realizations for 6 wind farm locations in Illinois and observations (dots) at nearest meteorological stations. Vertical lines represent beginning of day (12:00 AM).

Wind direction and speed samples required for our study are obtained from 
WRF. The WRF model [9] is a state-of-the-art numerical weather prediction system designed to serve both operational forecasting and atmospheric research needs. WRF is the result of a multi-agency and university effort to build a highly parallelizable code that can run across scales ranging from large-eddy to global simulations. The comprehensive description of the atmospheric physics includes cloud parameterization, land-surface models, atmosphere-ocean coupling, and broad radiation models.

We set up a computational nested domain structure for WRF including a high-resolution sector that covers the State of Illinois and two additional domains of larger coverage but lower resolution that provide boundary conditions for the nested domains [4]. The initial conditions from the assimilated state (also known as reanalyzed state) of the atmosphere are randomly perturbed and propagated in time through the nonlinear NWP model to obtain a set of ensembles that describe possible trajectories of the atmospheric conditions. The computational cost of this procedure is significant. Computing a single ensemble for Illinois over a 24-hour time window takes 6 hours of wall-clock time running on 32 processors. Because of these limitations, we computed only 30 ensembles for June 4, 2006 [4]. The ensembles have been validated using observations obtained from the National Climatic Data Center. The ensembles for six different wind farm locations are shown in Figure 3. The detailed procedures followed for the validation and a discussion of the possible sources of error that may make the confidence intervals presented here only a partial representation of the uncertainty are presented in [4].

Each ensemble provides the components of the wind velocity that are transformed to wind speed. This gives a 3D field in geographical coordinates (latitude, longitude, and elevation) evolving over time where the field points match the discretization mesh in the inner domain. The wind farm locations, however, do not match the discretization mesh. In addition, the typical hub height used for wind farms (80 meters) may also not match the WRF vertical layers. To remedy this issue, we use linear and bilinear interpolation to compute wind speeds at the farm locations from the WRF ensembles, therefore obtaining a set of 30 ensembles for the speeds at the desired 3D coordinates. We then compute the sample wind speed mean $\overline{\mathbf{x}}$, sample covariance $\mathbf{S}$, and the Rao-Blackwell-Ledoit-Wolf (RBLW) estimator $\widehat{\boldsymbol{\Sigma}}_{R B L W}$ as described in the next section. Using this approach, we can compute many wind speed scenarios by sampling a multivariate Gaussian with mean $\overline{\mathbf{x}}$ and estimated covariance matrix $\widehat{\Sigma}_{R B L W}$. In the end, the resulting wind speed samples are propagated through a typical power curve [4]. In the simulation of the Illinois network, this re- 
sampling is done for the next trading period (15 minutes) as required by the dispatch model (6a)-(6d).

Our computational framework is not restricted to the use of WRF and RBLW covariance estimators and can accommodate scenarios generated with different scenario generation techniques for wind speed, assuming that these can handle the large spatial coverage presented in our computational study. These scenario generation techniques include copula transformation $[10,11$, 12], probabilistic models [13], multivariate linear regression models [14], timerecursive covariance estimation [15], as well as the the method from [16] that uses autoregressive-moving-average model for the temporal correlations coupled with orthogonal transformations of the cross, spatial correlations. These approaches are purely statistical in nature and heavily rely on historical data, which is a salient difference from our NWP physics- and simulation-based approach. Finally, the statistical framework from [2] seems a very attractive alternative to NWP since it appears to improve the near-term (hour-ahead or 15 minutes-ahead) NWP-based forecasts; however, the spatial resolution of that work is considerably smaller than the one of the present work.

\subsection{Covariance Estimation Methods and Validation}

Since the number of WRF samples is smaller than the number of random variables (wind speeds at the wind farm locations over time), the sample covariance matrix is singular and cannot be used for sampling. The class of shrinkage estimators are known to be very robust in this case. They are also relatively inexpensive and can be used to capture spatial correlations over wide geographical areas. However, the performance of individual estimators in this class is highly dependent on the data set and underlying dynamics. In order to identify the best potential estimator for space-time wind correlations, we study the performance of the three most used shrinkage estimators on a the quasi-geostrophic model, which is representative of the atmospheric dynamics and can be cheaply simulated and sampled to obtain very accurate covariance matrices.

The problem of covariance estimation is computing the covariance matrix $\Sigma \in \mathbb{R}^{p \times p}$ of a random vector $\mathbf{y} \in \mathbb{R}^{p}$ based on a set of realizations or samples $\left\{\mathbf{x}_{i}\right\}_{i=1}^{n}$. Here $n$ is smaller than $p$. Let $\overline{\mathbf{x}}=\frac{1}{n} \cdot \sum_{i=1}^{n} \mathbf{x}_{i} \in \mathbb{R}^{p}$ denote the sample mean and $\mathbf{S}=\frac{1}{n} \cdot \boldsymbol{\Delta} \mathbf{X} \cdot \Delta \mathbf{X}^{T} \in \mathbb{R}^{p \times p}$ denote the sample covariance matrix, where $\Delta \mathbf{X}=\left[\mathbf{x}_{1}-\overline{\mathbf{x}}, \mathbf{x}_{2}-\overline{\mathbf{x}}, \ldots \mathbf{x}_{n}-\overline{\mathbf{x}}\right]$.

Covariance estimators for the case $p>n$ use regularizations of the sample

covariance matrix $\mathbf{S}$ to overcome the rank deficiency. One of the most common 
regularizations is the perturbation of the covariance matrix by a multiple of the identity, which leads to shrinkage estimators of the type

$$
\widehat{\mathbf{\Sigma}}=\alpha \cdot \mathbf{I}+\beta \cdot \mathbf{S}
$$

where $\mathbf{I} \in \mathbb{R}^{p \times p}$ is the identity matrix and $\alpha$ and $\beta$ are regularization parameters chosen to minimize the estimation error $\|\widehat{\Sigma}-\Sigma\|$, where $\|\cdot\|$ refers to the Frobenius norm. Since the real covariance matrix $\Sigma$ is unknown, different techniques are used to compute statistical estimates for $\alpha$ and $\beta$.

Ledoit and Wolf [17] propose the estimator

$$
\widehat{\mathbf{\Sigma}}_{L W}=\frac{b^{2}}{d^{2}} \cdot m \cdot \mathbf{I}+\frac{a^{2}}{d^{2}} \cdot \mathbf{S}
$$

where $m=\operatorname{tr}(\mathbf{S} \cdot \mathbf{I}) / p, a^{2}=d^{2}-b^{2}$,

$$
\begin{aligned}
d^{2} & =\operatorname{tr}\left([\mathbf{S}-m \cdot \mathbf{I}] \cdot[\mathbf{S}-m \cdot \mathbf{I}]^{\mathrm{t}}\right) / p, \text { and } \\
b^{2} & =\min \left(\frac{1}{n} \cdot \sum_{k=1}^{n} \frac{\operatorname{tr}\left(\left[\mathbf{x}_{k} \cdot \mathbf{x}_{k}^{\mathrm{t}}-\mathbf{S}\right] \cdot\left[\mathbf{x}_{k} \cdot \mathbf{x}_{k}^{\mathrm{t}}-\mathbf{S}\right]^{\mathrm{t}}\right)}{p}, d^{2}\right) .
\end{aligned}
$$

Here, $\operatorname{tr}(A)$ denotes the trace of matrix $A$.

Chen et al. [18] propose a refinement of the Ledoit-Wolf estimator, called the Rao-Blackwell Ledoit-Wolf estimator, which we denote by $\widehat{\Sigma}_{R B L W}$, that has better asymptotical properties (as $n \rightarrow \infty)$ than $\widehat{\Sigma}_{L W}$. This estimator is given by

$$
\begin{aligned}
\widehat{\mathbf{\Sigma}}_{R B L W} & =\rho_{R B L W} \cdot \mathbf{I}+\left(1-\rho_{R B L W}\right) \cdot \mathbf{S} \\
\rho_{R B L W} & =\min \left(\frac{\frac{n-2}{n} \cdot \operatorname{tr}\left(\mathbf{S}^{2}\right)+\operatorname{tr}^{2}(\mathbf{S})}{(n+2) \cdot\left[\operatorname{tr}\left(\mathbf{S}^{2}\right)-\frac{\operatorname{tr}^{2}(\mathbf{S})}{p}\right]}, 1\right) .
\end{aligned}
$$

A second estimator proposed in [18] is the oracle approximating shrinkage (OAS) estimator, which rely on a iterative procedure to provably converge to

$$
\begin{aligned}
\widehat{\mathbf{\Sigma}}_{O A S} & =\rho_{O A S} \cdot \mathbf{I}+\left(1-\rho_{O A S)}\right) \cdot \mathbf{S} \\
\rho_{O A S} & =\min \left(\frac{(1-2 / p) \cdot \operatorname{tr}\left(\mathbf{S}^{2}\right)+\operatorname{tr}^{2}(\mathbf{S})}{(n+1-2 / p)\left[\operatorname{tr}\left(\mathbf{S}^{2}\right)-\operatorname{tr}^{2}(\mathbf{S}) / p\right]}, 1\right) .
\end{aligned}
$$

The performance of these estimators is highly dependent on the data set. For example, $\widehat{\mathbf{\Sigma}}_{O A S}$ performs better than $\widehat{\mathbf{\Sigma}}_{R B L W}$ and $\widehat{\mathbf{\Sigma}}_{L W}$ for data coming from 
a fractional Brownian motion, but all three estimators perform comparably for Gaussian AR(1) processes (for small values of $n$ ) [18].

We investigate the performance of the three estimators using the lightweight quasi-geostrophic (QG) model, which is representative of a realistic atmospheric or oceanic data assimilating system [19]. We consider the QG model on the 2D Cartesian domain $\Omega=[0,1] \times[0,1]$ to estimate the covariance between $4 \times 4$ grid at 40 consecutive times $(p=160)$. The QG model describes the motion of a fluid and is mathematically expressed as $q_{t}=-\psi_{x}-\epsilon \cdot \mathcal{J}(\psi, q)-A \cdot \Delta^{3} \psi+$ $2 \cdot \pi \cdot \sin (2 \cdot \pi \cdot y)$, where $q$ is the potential vorticity, $\psi$ is the stream function, $\Delta$ is the Laplacian operator, $\mathcal{J}(\psi, q)=\psi_{x} \cdot q_{y}-\psi_{y} \cdot q_{x}$ is the Jacobian, and $x$ and $y$ are the horizontal and vertical components, respectively. The coefficients $A$ and $\epsilon$ are set to $2 \times 10^{-12}$ and $10^{-5}$, respectively.

The model uses homogeneous boundary conditions $\partial \psi=\partial q=0$ and initial conditions of the form

$$
\psi_{i, j}=\sin \left(\alpha+4 \beta x_{j} y_{i}\right)+\cos \left(\alpha+2 \beta x_{j} y_{i}\right)+\sin \left(\alpha+2 \beta x_{j} y_{i}\right) \cdot \cos \left(\alpha+4 \beta x_{j} y_{i}\right),
$$

where $\left(x_{i}, y_{j}\right), 1 \leq i, j \leq 64$, are the discretization points. Parameters $\alpha$ and $\beta$ describe the shift and amplitude waves, respectively.

Table 1: PRIAL norms for the estimators for the QG model

\begin{tabular}{c|c|c|c}
$n$ & $\delta\left(\boldsymbol{\Sigma}, \mathbf{S}, \widehat{\boldsymbol{\Sigma}}_{L W}\right)$ & $\delta\left(\boldsymbol{\Sigma}, \mathbf{S}, \widehat{\boldsymbol{\Sigma}}_{R B L W}\right)$ & $\delta\left(\boldsymbol{\Sigma}, \mathbf{S}, \widehat{\boldsymbol{\Sigma}}_{O A S}\right)$ \\
\hline 25 & $26 \%$ & $66 \%$ & $62 \%$ \\
40 & $35 \%$ & $64 \%$ & $61 \%$
\end{tabular}

The samples are built by picking four points from the discretization of $\Omega$ at 40 consecutive times and randomly perturbing the initial conditions using $\alpha=$ $1+\left|\mu_{1}\right|$ and $\beta=\mu_{2}$, where $\mu_{1}$ and $\mu_{2}$ are uniformly distributed random variables, $\mu_{1} \sim \mathcal{U}\left(0,10^{-4}\right)$ and $\mu_{2} \sim \mathcal{U}\left(0,10^{-2}\right)$. Each initial condition is propagated in time providing a sample associated with the 160 points of interest.

The quality of the estimators is inferred based on the percentage relative improvement in average loss norm (PRIAL) that describes how much an estimator $\widehat{\Sigma}$ improves the estimation of $\boldsymbol{\Sigma}$ with respect to S. PRIAL is defined as

$$
\delta(\boldsymbol{\Sigma}, \mathbf{S}, \widehat{\Sigma})=\frac{\mathbb{E}\left[\|\boldsymbol{\Sigma}-\mathbf{S}\|^{2}\right]-\mathbb{E}\left[\|\boldsymbol{\Sigma}-\widehat{\Sigma}\|^{2}\right]}{\mathbb{E}\left[\|\boldsymbol{\Sigma}-\mathbf{S}\|^{2}\right]}
$$



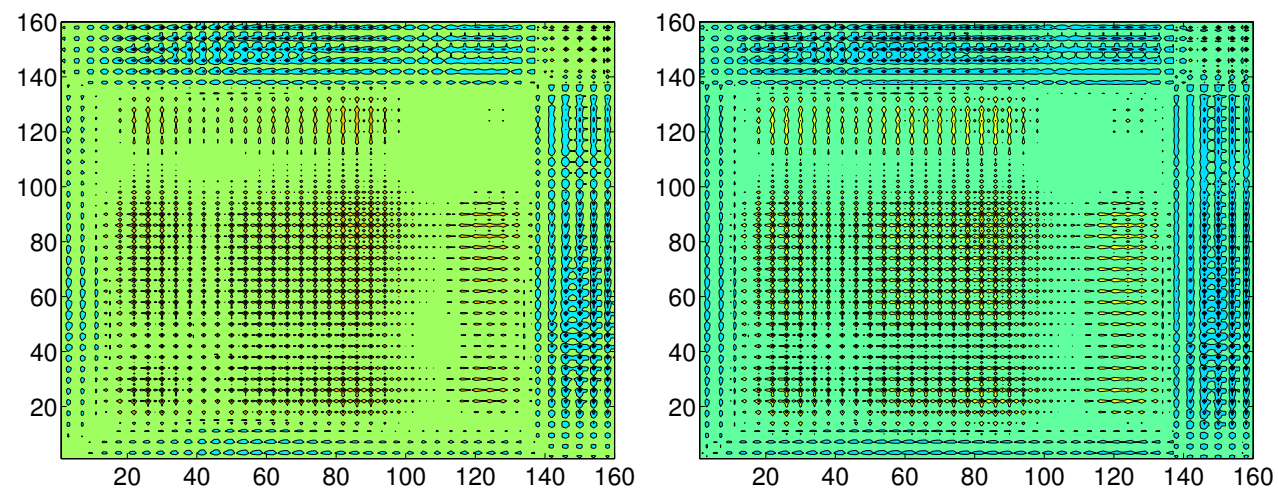

Figure 4: True covariance matrix (left) and RBLW estimator (right) for $n=25$.

We note that $\delta \in[0,1]$ and large values indicate good approximations of $\boldsymbol{\Sigma}$ by $\widehat{\boldsymbol{\Sigma}}$ relative to $\mathbf{S} ; \mathbf{S}$ itself gives $\delta=0$, whereas the perfect estimator $\boldsymbol{\Sigma}$ results in $\delta=1$. The PRIAL norms are shown in Table 1 .

We performed an asymptotic error analysis that revealed that 200 samples are enough for accurately calculating $\boldsymbol{\Sigma}$. The estimators are computed using $n=25$ and $n=40$ samples. We note that $\widehat{\Sigma}_{R B L W}$ offers the best improvement over $\mathbf{S}$ and is more robust for different number of samples. In Fig. 4 we show the structure of $\boldsymbol{\Sigma}$ and $\widehat{\boldsymbol{\Sigma}}_{R B L W}(n=25)$ and note that RBLW estimator preserves the structure of $\Sigma$.

\subsection{Economic Analysis}

To perform our benchmarks, we consider two strategies:

- (Corr): This strategy computes the forward dispatch solution using scenarios. This is done by using a distribution with covariance matrix $\widehat{\Sigma}_{R B L W}$. In other words, this strategy assumes that the ISO has correct correlation information.

- (Indep): This strategy computes the forward dispatch solution using scenarios that do not capture correlation. This is done by using a distribution with diagonal covariance matrix $\operatorname{diag}\left(\widehat{\boldsymbol{\Sigma}}_{R B L W}\right)$. We recall that the use of independent scenarios corresponds to the case in which each player submits to the ISO a set of scenarios created based on its own forecast, leaving the ISO without information about the correlation between players.

For both cases, we define the predicted cost and predicted decision as the optimal value and optimal decision vector of the stochastic dispatch problem under the corresponding sampling strategy. In addition, we define the realized 
cost as the cost of implementing the predicted decision under a "reference" sampling scheme, which we chose to be (Corr) with 256 scenarios. The realized costs are computed by first fixing the ahead predicted decision and then computing the corresponding dispatch cost under the reference sampling.

The sizes of the resulting optimization problems range from 14,635 decision variables and 12,884 constraints for $S=4$ to 763,579 decision variables and 704,372 constraints for $S=256$.

To solve these problems, we exploit the well-known dual-block angular structure using our open-source, parallel solver PIPS-IPM [20, 21, 22]. The ED model used in the numerical experiments can be found at [23] implemented in StochJuMP [24], a parallel, memory-distributed extension of the Julia package JuMP [25] for the specification of optimization models. While StochJuMP inherits from JuMP the easiness of expressing optimization models, it also offers additional syntax that allows distributing the large number of scenarios over a large number of computational nodes, circumventing the need to build and store the entire model in one node. StochJuMP is interfaced with PIPS-IPM using Julia's native support for calling $\mathrm{C}$ functions/callbacks. StochJump enables easy access to sophisticated computing platforms with minimal programming effort from the user.

We devised the simulations to also reveal the effect of the number of scenarios used on the dispatch cost. Moreover, because the dispatch cost is a random variable (it is an estimator), we needed to perform inference analysis. We use the approach proposed in [26] to compute the mean and standard deviation of the estimator. Here, for each $S=\{4,8,16,32,64,128,256\}$, we solve 256 batch instances in parallel (each instance uses its corresponding $S$ parallel processes). We highlight that this is an extremely computational expensive task. To perform this task we used the "Intrepid" IBM BG/P and "Mira" IBM BG/Q supercomputers at Argonne National Laboratory. The Intrepid supercomputer has 40 racks (40,960 nodes); each node has a $850 \mathrm{MHz}$ quad-core PowerPC processor. Mira has 48 racks, each of 1024 nodes. Each node has 16 PowerPC A2 cores operating at 1,600 Mhz. In our simulations we have used up to 16,384 nodes on each system. On Mira, the time for solving 256 batches is between 6 minutes (for $S=4$ ) and 8 minutes (for $S=256$ ). The slight increase in the execution times with $S$ is primarily caused by $I / O$ overhead. Times required to perform all of these expensive simulations in serial would be on the order of days. We thus highlight the importance of leveraging high-performance computing capabilities to enable high-resolution analyzes. 


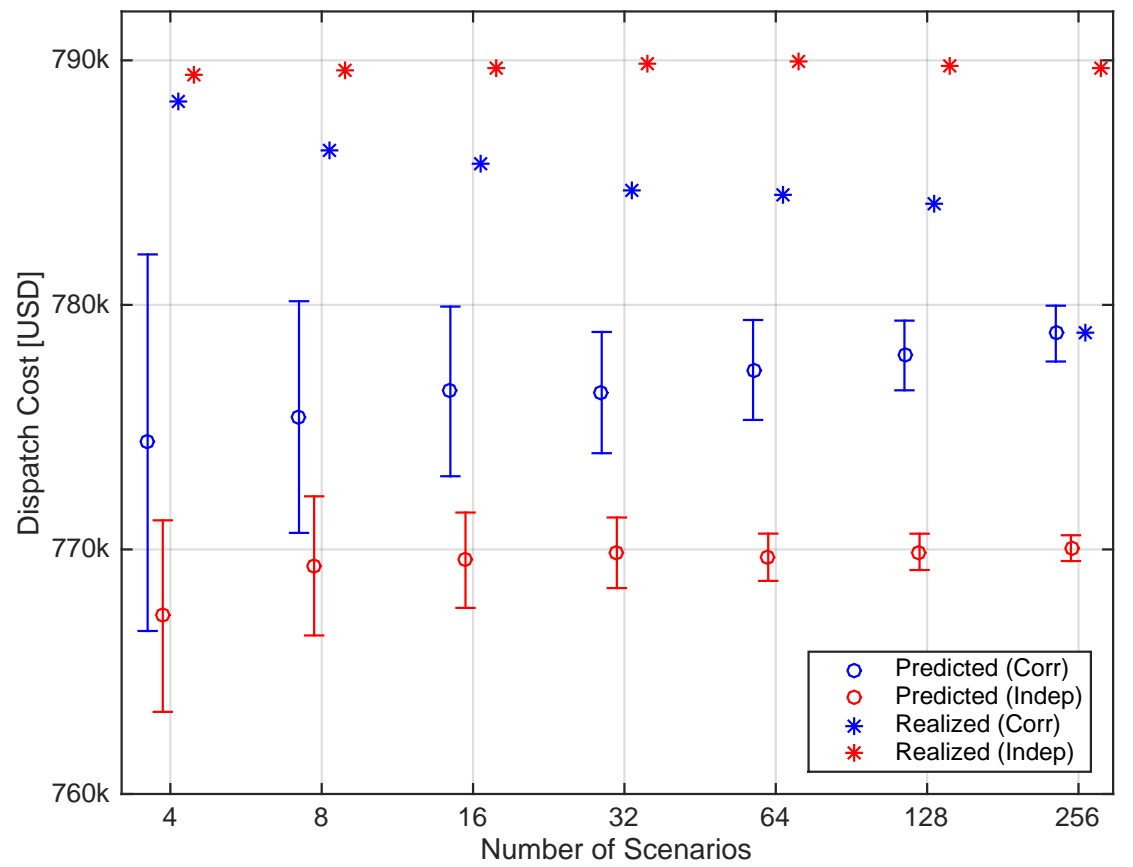

Figure 5: Mean and 95\% confidence intervals for dispatch cost predicted with $S=$ $\{4,8,16,32,64,128,256\}$ correlated and independent scenarios based on 256 batches. We also show "realized" dispatch costs, corresponding to costs obtained by implementing the predicted dispatch decisions under the correlated scenarios sampling scheme. 
Our results are summarized in Fig. 5 and lead to several relevant observations:

- A dispatch cost gap exists between the realized dispatch costs of the (Corr) and (Indep) strategies of about $10,000 \mathrm{USD} / \mathrm{hr}$ or $1.5 \%$. If this error is extrapolated over a year, it would result in a gap of nearly 100 million USD/yr. This gap is interpreted as an operational error induced by the suboptimality of the dispatch decisions due to the use of an incorrect characterization of the atmospheric conditions and of the associated wind power levels when clearing the market. We also note that this gap does not close as the number of scenarios increases, being consistent with the analytical dispatch model of Section 2.

- The (Indep) and (Corr) strategies exhibit different error bands, and the bands converge at significantly different rates. For the (Corr) strategy, the error bands are small for 64 or more scenarios. In particular, the standard deviation of the dispatch cost is $0.45 \%$ and $0.36 \%$ when using 128 and 256 scenarios, respectively. The difference in the error bands indicates that neglecting correlation can have important effects on the stability of the dispatch cost as might led the practitioner to believe that it requires fewer samples of the atmospheric conditions than actually necessary.

- The gap between the predicted and realized costs of (Indep) is nearly 20,000 USD/hr and does not close. This gap should be interpreted as a prediction error induced by improperly characterizing the uncertainty of the atmospheric conditions (ignoring correlations).

- The gap between the predicted cost of the (Corr) strategy and the predicted cost of the (Indep) strategy reflect the economic impact of operating the grid under correlated and uncorrelated wind conditions. Consequently, the proposed computing framework can also be used to assess how system costs are affected by different correlation levels at different geographical locations.

- Even under the (Corr) sampling, a gap occurs between the predicted and realized dispatch costs, as shown in Fig. 5 by the blue graphs. This gap diminishes on expectation as the number of scenarios increases since more accurate uncertainty characterizations are employed, as the result of using an increasing number of scenarios. In stochastic optimization parlance, this is known as the value of stochastic solution [27]. For 256 scenarios, 
the expectation of the realized dispatch costs matches the predicted cost, which is the result of using the same (Corr) distribution, approximated using on 256 scenarios, both for realized and predicted costs. Ideally one should use the "true" distribution for wind speeds as the reference distribution in the inference analysis of Fig. 5; however, this is not possible since this distribution is unknown and can only be approximated (by (re)sampling). This is the reason we used the distribution given by 256 scenarios as the reference, "true" distribution. In practice, the operator may choose the "true" (Corr) sampling distribution based on the financial (dispatch cost) accuracy he or she needs to satisfy under wind uncertainty. For example, the (Corr) 256-scenario distribution gives a 95\% CI of less than $2000 \mathrm{USD} / \mathrm{hr}(0.26 \%)$ bandwidth.

Our results indicate that a coordinated weather forecasting capability is needed to create proper uncertainty characterizations of atmospheric conditions. In other words, a coordinated forecast that captures long-range correlations should be used to generate scenarios of atmospheric conditions (e.g., wind speed and direction). The suppliers can then use these scenarios to generate wind power scenarios that capture their local conditions and private knowledge of their wind farms. On the contrary, if suppliers are allowed to provide their own weather forecasts that only capture local conditions, cost and price biases will arise.

We also note that if no correlation is captured, the dispatch system will be more conservative and dispatch more expensive thermal supply as opposed to wind supply so that overall the system will be less cost and environmentally efficient, ultimately affecting consumers and wind suppliers. By better capturing correlations the operator faces less risk and more wind can potentially be dispatched.

\section{Conclusions}

We have presented a high-performance computing framework to demonstrate that neglecting correlations between multiple wind supply points can result in strong biases of dispatch cost. Our conclusions are drawn from a detailed study that incorporates high-resolution wind speed forecasts from numerical weather prediction models, robust and computationally cheap covariance estimation technique capable of capturing spatio-temporal correlations for wide geographical areas, and stochastic dispatch models. We believe that the results are of relevance as they suggest that a coordinated uncertainty characterization procedure is needed to capture long-range correlation. We demonstrate that 
the use of parallel computing enables us to perform sophisticated, system-wide analyzes in minutes (as opposed to days if computations would be performed in serial).

\section{Appendix A. Proof of Proposition 1.}

We start with an intermediary result.

Lemma 1. $\mathbb{E}[X \mid X \leq d]=-\sigma^{2} \phi(d)+\mu \Phi(d)$.

Proof. By direct computation we have

$$
\begin{aligned}
& \mathbb{E}[X \mid X \leq d]=\int_{-\infty}^{d} x \phi(x) d x=-\frac{\sigma}{\sqrt{2 \pi}} \int_{-\infty}^{d}\left(-\frac{x-\mu}{\sigma^{2}}-\frac{\mu}{\sigma^{2}}\right) \exp \left(-\frac{(x-\mu)^{2}}{2 \sigma^{2}}\right) d x \\
& =-\left.\frac{\sigma}{\sqrt{2 \pi}} \exp \left(-\frac{(x-\mu)^{2}}{2 \sigma^{2}}\right)\right|_{\infty} ^{d}+\frac{\mu}{\sqrt{2 \pi} \sigma} \int_{-\infty}^{d} \exp \left(-\frac{(x-\mu)^{2}}{2 \sigma^{2}}\right) d x \\
& =-\sigma^{2} \phi(d)+\mu \Phi(d) .
\end{aligned}
$$

Proof of Proposition 1. We first show that $c_{d}(\sigma)$ given by (5) is strictly increasing. For this let $p(x, \sigma)=-\frac{(x-\mu)^{2}}{2 \sigma^{2}}$; therefore $\phi(x, \sigma)=\frac{1}{\sigma \sqrt{2 \pi}} \exp (p(x, \sigma))$. Observe that

$$
\begin{aligned}
\frac{d}{d \sigma} p(x, \sigma) & =+\frac{(x-\mu)^{2}}{\sigma^{3}}, \quad \frac{d}{d x} p(x, \sigma)=-\frac{(x-\mu)}{\sigma^{2}}, \\
\frac{d}{d x} \phi(x, \sigma) & =\phi(x, \sigma) \cdot \frac{d}{d x} p(x, \sigma), \\
\frac{d}{d \sigma} \phi(x, \sigma) & =\phi(x, \sigma) \cdot \frac{d}{d \sigma} p(x, \sigma)-\frac{1}{\sigma^{2} \sqrt{2 \pi}} \exp (p(x, \sigma)) \\
& =\frac{(x-\mu)^{2}}{\sigma^{3}} \cdot \phi(x, \sigma)-\frac{1}{\sigma} \phi(x, \sigma) .
\end{aligned}
$$


The derivative of $\Phi$ with respect to $\sigma$ is computed as follows:

$$
\begin{aligned}
& \frac{d}{d \sigma} \Phi(d, \sigma)=\frac{d}{d \sigma} \int_{-\infty}^{d} \phi(p(x, \sigma)) d x=\int_{-\infty}^{d} \frac{d}{d \sigma} \phi(p(x, \sigma)) d x \\
& \stackrel{(\mathrm{A} .3)}{=} \int_{-\infty}^{d} \frac{(x-\mu)^{2}}{\sigma^{3}} \cdot \phi(p(x, \sigma)) d x-\frac{1}{\sigma} \int_{-\infty}^{d} \phi(p(x, \sigma)) d x \\
& =-\int_{-\infty}^{d} \phi(p(x, \sigma)) \cdot\left(-\frac{x-\mu}{\sigma^{2}}\right) \cdot \frac{x-\mu}{\sigma} d x-\frac{1}{\sigma} \Phi(d, \sigma) \\
& \stackrel{(\text { A.1) }}{=}-\int_{-\infty}^{d} \phi(p(x, \sigma)) \cdot \frac{d}{d x} p(x, \sigma) \cdot \frac{x-\mu}{\sigma} d x-\frac{1}{\sigma} \Phi(d, \sigma) \\
& \stackrel{(\text { A.2 })}{=}-\int_{-\infty}^{d} \frac{d}{d x} \phi(p(x, \sigma)) \cdot \frac{x-\mu}{\sigma} d x-\frac{1}{\sigma} \Phi(d, \sigma) \\
& =-\left.\phi(p(x, \sigma)) \cdot \frac{x-\mu}{\sigma}\right|_{-\infty} ^{d}+\frac{1}{\sigma} \int_{-\infty}^{d} \phi(p(x, \sigma)) d x-\frac{1}{\sigma} \Phi(d, \sigma) \\
& =-\frac{d-\mu}{\sigma} \cdot \phi(p(d, \sigma)) .
\end{aligned}
$$

By differentiating (5) and using (A.3) and (A.4), we compute

$$
\begin{aligned}
\frac{d}{d \sigma} c_{d}(\sigma) & =\left(p_{t h}-p_{w}\right)\left((d-\mu) \frac{d}{d \sigma} \Phi(d, \sigma)+2 \sigma \phi(x, d)+\sigma^{2} \frac{d}{d \sigma} \phi(d, \sigma)\right) \\
& =\left(p_{t h}-p_{w}\right)\left(-\frac{(d-\mu)^{2}}{\sigma} \cdot \phi(d, \sigma)+2 \sigma \phi(d, \sigma)+\frac{(d-\mu)^{2}}{\sigma} \cdot \phi(d, \sigma)-\sigma \phi(d, \sigma)\right) \\
& =\sigma\left(p_{t h}-p_{w}\right) \phi(d, \sigma)>0,
\end{aligned}
$$

which shows that $c_{d}(\sigma)$ is strictly increasing. Since $\sigma(\rho)=\sqrt{\sigma_{1}^{2}+2 \rho \sigma_{1} \sigma_{2}+\sigma_{2}^{2}}$ is a strictly increasing function of $\rho$, we conclude that $c_{d}$ is also strictly increasing function of $\rho$.

\section{Acknowledgments}

This work was supported by the U.S. Department of Energy, under Contract No. DE-AC02-06CH11357 and used resources of the Argonne Leadership Computing Facility at Argonne National Laboratory.

[1] G. Pritchard, G. Zakeri, A. Philpott, A single-settlement, energy-only electric power market for unpredictable and intermittent participants, Operations Research 58 (4-2) (2010) 1210-1219. 
[2] L. Xie, Y. Gu, X. Zhu, M. Genton, Short-term spatio-temporal wind power forecast in robust look-ahead power system dispatch, Smart Grid, IEEE Transactions on 5 (1) (2014) 511-520. doi:10.1109/TSG.2013.2282300.

[3] M. Ghofrani, A. Arabali, M. Etezadi-Amoli, Y. Baghzouz, Operating reserve requirements in a power system with dispersed wind generation, in: Innovative Smart Grid Technologies, IEEE PES, 2012, pp. 1-8. doi:10.1109/ISGT.2012.6175804.

[4] E. Constantinescu, V. Zavala, M. Rocklin, S. Lee, M. Anitescu, A computational framework for uncertainty quantification and stochastic optimization in unit commitment with wind power generation, IEEE Transactions on Power Systems 26 (1) (2011) 431-441.

[5] V. M. Zavala, J. Birge, M. Anitescu, A stochastic market clearing formulation with consistent pricing properties, Preprint ANL/MCS-P5110-0314.

[6] X. Fang, F. Li, Y. Wei, R. Azim, Y. Xu, Reactive power planning under high penetration of wind energy using benders decomposition, IET Generation, Transmission \& Distribution 9 (14) (2015) 1835-1844.

[7] R. Arjmand, M. Rahimiyan, Impact of spatio-temporal correlation of wind production on clearing outcomes of a competitive pool market, Renewable Energy 86 (2016) 216-227.

[8] Y. Wei, F. Li, K. Tomsovic, Measuring the volatility of wholesale electricity prices caused by wind power uncertainty with a correlation model, IET Renewable Power Generation 6 (5) (2012) 315-323.

[9] W. Skamarock, J. Klemp, J. Dudhia, D. Gill, D. Barker, M. Duda, X.Y. Huang, W. Wang, J. Powers, A description of the Advanced Research WRF version 3, Tech. Rep. Tech Notes-475+ STR, NCAR (2008).

[10] G. Papaefthymiou, D. Kurowicka, Using copulas for modeling stochastic dependence in power system uncertainty analysis, Power Systems, IEEE Transactions on 24 (1) (2009) 40-49. doi:10.1109/TPWRS.2008.2004728.

[11] J. Tastu, P. Pinson, H. Madsen, Space-time trajectories of wind power generation: Parameterized precision matrices under a Gaussian copula approach, in: Lecture Notes in Statistics: Modeling and Stochastic Learning for Forecasting in High Dimension, in press, 2014. 
[12] M. Wytock, J. Kolter, Large-scale probabilistic forecasting in energy systems using sparse Gaussian conditional random fields, in: IEEE 52nd Annual Conference on Decision and Control, 2013, pp. 1019-1024.

[13] J. Sumaili, H. Keko, V. Miranda, Z. Zhou, A. Botterud, J. Wang, Finding representative wind power scenarios and their probabilities for stochastic models, in: 16th International Conference on Intelligent System Application to Power Systems, 2011, pp. 1-6.

[14] G. Yang, H. Chen, K. Zhang, Y. Wang, F. Xu, Modelling and design of wind power forecast error estimation system, in: Advanced Research and Technology in Industry Applications (WARTIA), 2014 IEEE Workshop on, 2014, pp. 1320-1323. doi:10.1109/WARTIA.2014.6976526.

[15] P. Pinson, H. Madsen, H. A. Nielsen, G. Papaefthymiou, B. Klöckl, From probabilistic forecasts to statistical scenarios of short-term wind power production, Wind Energy 12 (1) (2009) 51-62.

[16] J. Morales, R. Mínguez, A. Conejo, A methodology to generate statistically dependent wind speed scenarios, Applied Energy 87 (3) (2010) 843-855.

[17] O. Ledoit, M. Wolf, A well-conditioned estimator for large-dimensional covariance matrices, Journal of Multivariate Analysis 88 (2) (2004) 365411.

[18] Y. Chen, A. Wiesel, A. Hero, Shrinkage estimation of high dimensional covariance matrices, in: IEEE International Conference on Acoustics, Speech and Signal Processing, 2009, pp. 2937-2940.

[19] P. Sakov, P. R. Oke, A deterministic formulation of the ensemble Kalman filter: An alternative to ensemble square root filters, Tellus A 60 (2) (2008) 361-371.

[20] M. Lubin, C. G. Petra, M. Anitescu, V. Zavala, Scalable stochastic optimization of complex energy systems, in: Proceedings of 2011 International Conference for High Performance Computing, Networking, Storage and Analysis, SC '11, ACM, New York, USA, 2011, pp. 64:1-64:64.

[21] C. G. Petra, M. Anitescu, A preconditioning technique for Schur complement systems arising in stochastic optimization, Computational Optimization and Applications 52 (2012) 315-344. 
[22] C. G. Petra, O. Schenk, M. Lubin, K. Gärtner, An augmented incomplete factorization approach for computing the Schur complement in stochastic optimization, SIAM Journal on Scientific Computing 36 (2) (2014) C139C162.

[23] StochJuMP: a modeling environment for stochastic optimization on highperformance computing platforms (August 2014).

URL https://github.com/joehuchette/StochJuMP.jl

[24] J. Huchette, M. Lubin, C. Petra, Parallel algebraic modeling for stochastic optimization, in: Proceedings of the 1st First Workshop for High Performance Technical Computing in Dynamic Languages, HPTCDL '14, IEEE Press, Piscataway, NJ, USA, 2014, pp. 29-35.

[25] I. Dunning, J. Huchette, M. Lubin, JuMP: A modeling language for mathematical optimization, Optimization-online.org.

[26] J. Linderoth, A. Shapiro, S. Wright, The empirical behavior of sampling methods for stochastic programming, Annals of Operations Research 142 (1) (2006) 215-241.

[27] J. R. Birge, F. Louveaux, Introduction to stochastic programming, Springer-Verlag, New York,, 1997. 